\title{
Percepções de mães de pacientes sobre o atendimento odontológico na Clínica de odontopediatria da Escola Bahiana de Medicina e Saúde Pública
}

\author{
Perceptions of Mothers of patients on Dental Care Clinic In Paediatric \\ Dentistry Bahian School of medicine and Public Health
}

Tatiana Frederico de Almeida*

Thiago de Souza Azevedo**

Flávia Godinho Costa Wanderley ${ }^{* * *}$

Elisabeth Martinez Fonseca***

\section{Resumo}

Ao lado dos experimentos técnicos da Odontologia, é necessário que pesquisas sobre satisfação dos pacientes contribuam para o avanço do saber e dos serviços de saúde, especialmente no grupo infanto-juvenil, o qual se encontra em uma etapa da vida muito importante na determinação da qualidade de vida relacionada com a saúde bucal. Objetivo: a presente pesquisa objetiva descrever as percepções de mães sobre o atendimento odontológico prestado pelo componente curricular de odontopediatria na Clínica da Criança II da Escola Bahiana de Medicina e Saúde Pública (EBMSP). Sujeitos e métodos: este é um estudo de caráter qualitativo realizado com as mães das crianças atendidas no ambulatório da EBMSP. A amostra foi de conveniência: dez mães foram entrevistadas individualmente até se atingir um ponto de saturação nas entrevistas, segundo um roteiro semi-estruturado, o qual abordou questões sobre a qualidade dos serviços oferecidos, de modo a identificar suas potencialidades e fragilidades, bem como as habilidades do dentista em formação mais valorizadas durante o atendimento. Resultados: aspectos cognitivos, afetivos e psicomotores foram destacados no atendimento, embora os psicossociais tenham se mostrado mais valorizados pelas mães. Problemas no acolhimen- to foram caracterizados pela dificuldade na marcação de consulta e a falta de pontualidade dos estudantes. Conclusões: as entrevistadas declararam como dentista ideal aquele que reune diferentes habilidades, além das técnicas. A pesquisa apontou que as percepções de atendimento ambulatorial podem contribuir para a melhoria da qualidade dos serviços oferecidos, assim como da saúde bucal da população assistida.

Palavras-chave: Atendimento odontológico. Crianças. Odontopediatria.

\section{Introdução}

Mesmo com o avanço constante das técnicas na Odontologia, alguns pacientes, principalmente as crianças, mantêm uma imagem negativa da assistência odontológica, desenvolvendo comportamento de medo do dentista. Isso demonstra que o conhecimento dos fatores psicossociais e o controle desse sentimento pelos profissionais que atuam na odon-

Doutora em Saúde Coletiva pela Universidade Federal da Bahia, professora adjunta do Curso de Odontologia da Escola Bahiana de Medicina e Saúde Pública, Salvador, Bahia, Brasil.

Graduado em Odontologia pela Escola Bahiana de Medicina e Saúde Pública, Salvador, Bahia, Brasil.

* Estudante de Iniciação Científica da Escola Bahiana de Medicina e Saúde Pública, Salvador, Bahia, Brasil/ Bolsista FAPESB e integrante voluntária do Programa de Educação Tutorial PET-ODONTO-BAHIANA.

**** Estudante da Escola Bahiana de Medicina e Saúde Pública, Salvador, Bahia, Brasil/ Bolsista do Programa de Educação Tutorial PET-ODONTO-BAHIANA. 
topediatria é tão importante quanto o emprego do saber técnico. Para evitar as imagens negativas e outros problemas não fisiológicos do atendimento, é fundamental a aplicação de pesquisas qualitativas que busquem compreender a imagem formada nos indivíduos que são atendidos ${ }^{1}$.

No caso das crianças, a pesquisa de campo, recolhida diretamente junto a elas, torna-se dificultada, tendo em vista que é natural que a timidez ou a imaturidade afetem a emissão dos dados, considerando que o pesquisador representa para elas um estranho. Além disso, nessa faixa de idade, é natural que a compreensão de alguns termos e condições da saúde sejam ainda desconhecidos.

Dessa forma, é mais viável que as mães sejam consultadas na condição de intermediárias e, também, de participantes secundárias do tratamento, pois elas realizam comunicação íntima com as crianças, no período pós-atendimento, e, na maioria das vezes, durante o atendimento. Cardoso et al. ${ }^{2}$ (2007), embora abordem exclusivamente o atendimento médico e não o odontológico, consideram que a satisfação dos pais é um fator preponderante na promoção da saúde infantil, uma vez que eles são os mediadores entre o profissional da saúde e a criança, não só por acompanharem as consultas, mas também por serem os principais responsáveis pela aplicação das recomendações e dos tratamentos que os profissionais indicam. Essas considerações são perfeitamente cabíveis para a odontopediatria, visto que demonstram como as informações apresentadas pelas mães devem constituir as bases para que seja possível entender os impactos psicossociais da intervenção dos dentistas pediátricos, afinal a infância contém processos que só por meio dos pais é possível intervir. Assim, as mães dos pacientes devem estar satisfeitas e informadas sobre as ações relativas ao tratamento para que haja efetividade dos procedimentos realizados pelo odontopediatra ${ }^{3}$.

Dessa forma, segundo Silva e Muniz ${ }^{3}$ (2007), se as medidas de higiene bucal e de controle da dieta forem adotadas corretamente, doenças como a cárie e a doença periodontal terão seu índice de prevenção aumentado. Assim, a cooperação e a instrução das mães, que são as principais educadoras nos lares, emergem como elementos fundamentais, desde os primeiros anos da criança, para que hábitos saudáveis sejam mantidos por toda a vida ${ }^{4}$. Uma vez que esses fatores psicossociais são de primeira ordem para o sucesso do atendimento odontopediátrico, é indispensável que as mães sejam colaboradoras ativas durante o tratamento ${ }^{4}$. Robles et al. ${ }^{4}(2008)$ relatam que a preocupação maior das mães não é somente com a aparência dos dentes e com alguns possíveis problemas funcionais, devido à perda de unidades dentárias, pois alterações causadas pela doença cárie provocam não só prejuízos na mastigação e limitações que envolvem o falar e o sorrir como, também, estigmatização das crianças nos mais diversos meios sociais, o que constitui um fator determinante na construção da autoestima ao longo de toda a vida. Esses autores evidenciaram que as mães que acompanharam seus filhos ao ambulatório de odontopediatria da UFSC valorizaram, no trabalho do Odontopediatra, alguns elementos importantes, tais como afetividade, qualificação, prazer em realizar sua profissão, capacidade de informar os procedimentos, entre outras ${ }^{5}$. Este estudo tem como propósito avaliar as percepções das mães quanto ao atendimento prestado na Clínica de odontopediatria da Escola Bahiana de Medicina e Saúde Pública (EBMSP), sob a perspectiva da qualidade dos serviços oferecidos, por meio de um estudo com abordagem qualitativa. Dessa maneira, também se pretende identificar os potenciais e as fragilidades do atendimento nessa clínica pertencente a essa Instituição de Ensino Superior (IES). Esta pesquisa insere-se no contexto de necessidade de avaliação dos aspectos não fisiológicos e estruturados dos tratamentos odontológicos nas crianças. Como não há muitos estudos de casos correlatos, a presente pesquisa fornece subsídios teóricos para uma maior compreensão da problemática. Pretende-se, com os resultados desta pesquisa, enriquecer a literatura com uma temática que passa despercebida por muitos profissionais, apesar de sua grande importância para que seja possível entender os comportamentos e a influência que os processos de promoção da saúde e de prevenção da doença exercem na qualidade de vida das pessoas. Isso permitirá a melhoria da qualidade do atendimento oferecido na EBMSP a esses pacientes, na medida em que os resultados deste estudo forem divulgados entre os cirurgiões-dentistas em formação.

\section{Sujeitos e métodos}

Foi realizada uma pesquisa de campo de caráter qualitativo, através de entrevistas individuais no segundo semestre letivo de 2012. A amostra total foi de dez participantes, todas mães de crianças atendidas no ambulatório de odontopediatria da EBMP. A seleção ocorreu de forma intencional, ou seja, mães de pacientes que aguardavam a consulta dos seus filhos foram convidadas a participar do estudo. Uma condição necessária para participação na entrevista, o critério fundamental foi que elas já tivessem acompanhado seu filho, no mínimo, em uma consulta odontológica realizada na EBMSP. As entrevistas foram realizadas por um estudante do $9^{\circ}$ semestre do curso de graduação em Odontologia da EBMSP. Inicialmente, foram coletados dados de identificação das mães (iniciais do nome, idade, número de filhos, idade dos filhos, estado civil, profissão, escolaridade, renda familiar, data do início do tratamento na EBMSP e motivo da consulta). $\mathrm{O}$ roteiro semi-estruturado (Quadro 1) serviu como base para a entrevista individual, em que se tratou das percepções das mães sobre o serviço de odontopediatria da EBMSP (potencialidades, fragilida- 
des), e também foram questionadas a respeito das características que os dentistas apresentam ou deveriam manifestar durante o atendimento (dentista ideal). Ao realizar a análise de conteúdo dos dados produzidos, tais aspectos foram considerados como categorias analíticas deste estudo. Este estudo foi conduzido de acordo com os preceitos determinados pela resolução 196/88, do Conselho Nacional de Saú- de, do Ministério da Saúde ${ }^{6}$, e pela resolução CFO 118/2012, do Código de Ética Profissional Odontológico $^{7}$. As mães tiveram a reserva do anonimato garantida, e as entrevistas foram gravadas e transcritas, e a identificação das mães se deu por pseudônimos nas citações. Este estudo foi submetido e aprovado pelo Comitê de Ética em Pesquisa da EBMSP por meio do protocolo n. 06394812.6.0000.5544.

Quadro 1 - Questionário da entrevista

Data __ $1 \ldots 1$

Qual o nome completo da senhora?

Qual a sua idade?

Quantos filhos a senhora tem?

Qual é a idade dele(as)?

Qual é o estado civil da senhora?

Qual é a profissão da senhora?

Qual a escolaridade da senhora?

A renda familiar da senhora está em torno de quantos reais?

Quando foi o início do tratamento do seu filho aqui na faculdade?

Qual foi o motivo da primeira consulta?

Como a senhora qualifica o atendimento oferecido aqui, em relação à sua satisfação e à do seu(s) filho(s)?

E o filho da senhora, o que ele fala a respeito do atendimento?

E eles ficam alegres no dia da consulta?

Quais são os pontos positivos e os negativos do atendimento?

Quais características a senhora acredita que um dentista ideal, ou seja, o dentista que oferecesse o melhor tratamento possível ao seu(s) filho(s), deveria ter?

\section{Resultados}

Participaram deste estudo dez mães de crianças, que foram atendidas no ambulatório de odontopediatria da EBMSP em 2012. Primeiramente, os dados das mães foram tratados quantitativamente e, em seguida, qualitativamente. Desse total, a maioria era dona de casa $40 \%$, enquanto que $30 \%$ realizavam a atividades doméstica fora do lar. Quanto à escolaridade, $30 \%$ apresentavam ensino fundamen- tal incompleto, a mesma porcentagem tinha completado o ensino médio. Nenhuma mãe apresentava ensino superior completo ou em curso. A renda familiar variou bastante, entre 332 reais mensais até 2000 reais mensais, porém 50\% das mães recebiam o valor aproximado de um salário mínimo. A quantidade de filhos variou entre um e quatro. Em relação ao motivo da busca de tratamento na EBMSP, $60 \%$ das entrevistadas manifestaram ter seguido a indicação da creche (Tab. 1). 
Tabela 1 - Dados socioeconômicos combinados aos dados do tratamento das mães de pacientes entrevistadas. EBMSP, Salvador - BA, 2012

\begin{tabular}{|c|c|c|c|c|c|c|c|c|c|}
\hline Entrevistada & Idade & Ocupação & $\begin{array}{l}\text { Estado } \\
\text { civil }\end{array}$ & Escolaridade & $\begin{array}{l}\text { Renda } \\
\text { familiar }\end{array}$ & $\begin{array}{l}\text { Número } \\
\text { de filhos }\end{array}$ & $\begin{array}{l}\text { Idade dos } \\
\text { filhos (em } \\
\text { anos) }\end{array}$ & $\begin{array}{l}\text { Início do } \\
\text { primeiro } \\
\text { tratamento }\end{array}$ & $\begin{array}{l}\text { Motivo da primeira } \\
\text { consulta do primeiro } \\
\text { filho consultado }\end{array}$ \\
\hline S.R.R & 33 & Dona de casa & Casada & $\begin{array}{l}\text { Médio } \\
\text { Completo }\end{array}$ & 2.000 & 1 & 3 & 2012 & $\begin{array}{l}\text { Dente quebrado na } \\
\text { frente }\end{array}$ \\
\hline A.M.S & 35 & Dona de casa & Casada & $\begin{array}{l}\text { Médio } \\
\text { Completo }\end{array}$ & 2.000 & 4 & $14,9,7,3$ & 2007 & $\begin{array}{l}\text { Cárie de mamadeira } \\
\text { para simplificar, teve } \\
\text { outros traumas }\end{array}$ \\
\hline $\mathrm{P}$ & 29 & Dona de casa & Casada & $\begin{array}{l}\text { Fundamental } \\
\text { Incompleto }\end{array}$ & 700 & 3 & $9,5,6$ & 2011 & $\begin{array}{l}\text { Recomendação da } \\
\text { creche - restauração }\end{array}$ \\
\hline $\mathrm{N}$ & 35 & Desempregada & Casada & $\begin{array}{l}\text { Fundamental } \\
\text { Completo }\end{array}$ & $\begin{array}{l}\text { Menor } \\
\text { que } 700\end{array}$ & 4 & $19,15,10,7$ & 2012 & $\begin{array}{l}\text { Má formação da } \\
\text { gengiva }\end{array}$ \\
\hline M & 45 & Doméstica & Solteira & $\begin{array}{l}\text { Fundamental } \\
\text { Incompleto }\end{array}$ & 700 & 1 & 7 & 2011 & $\begin{array}{l}\text { Recomendação da } \\
\text { escola }\end{array}$ \\
\hline MFM & 47 & Dona de casa & Casada & $\begin{array}{l}\text { Fundamental } \\
\text { Completo }\end{array}$ & 700 & 2 & 14,12 & 2012 & $\begin{array}{l}\text { Recomendação da } \\
\text { creche }\end{array}$ \\
\hline CGSS & 36 & $\begin{array}{l}\text { Assistente de } \\
\text { serviços gerais }\end{array}$ & Solteira & $\begin{array}{l}\text { Médio em } \\
\text { curso }\end{array}$ & 700 & 2 & 14,16 & 2000 & $\begin{array}{l}\text { Recomendação da } \\
\text { creche }\end{array}$ \\
\hline MCT & 34 & Lavadora & Solteira & $\begin{array}{l}\text { Fundamental } \\
\text { Incompleto }\end{array}$ & 332 & 3 & $11,5,2$ & 2010 & $\begin{array}{l}\text { Excesso no dente que } \\
\text { criava bolha e saia } \\
\text { secreção e ficava } \\
\text { incomodando }\end{array}$ \\
\hline RGA & 58 & Doméstica & Casada & $\begin{array}{l}\text { Médio } \\
\text { Incompleto }\end{array}$ & 700 & 2 & 14,15 & 2001 & $\begin{array}{l}\text { Recomendação da } \\
\text { creche }\end{array}$ \\
\hline JCS & 26 & Doméstica & Solteira & $\begin{array}{l}\text { Fundamental } \\
\text { Completo }\end{array}$ & 1400 & 2 & 13,2 & 2005 & Cárie \\
\hline
\end{tabular}

Os resultados deste estudo qualitativo foram analisados, a partir de três categorias principais: potencialidades (pontos positivos do atendimento); fragilidades no atendimento (pontos negativos) e o dentista ideal.

\section{Pontos positivos no atendimento}

$\mathrm{O}$ tema relacionamento dentista-paciente foi um tema muito abordado durante as entrevistas, mostrando a importância dada pelas mães ao carinho e ao afeto presentes no contato entre o profissional e a criança. Esse depoimento constitui um indicador da importância da construção de um relacionamento pautado na empatia entre as partes envolvidas:

Os dentistas são receptivos, são educados, tem uma boa comunicação que, mesmo não nos conhecendo fora daqui, parece que, aqui dentro, todo mundo é amigo, a gente conversa, todo mundo é cordial, tem um atendimento, não só profissional, mas a gente acaba se tornando amigo, né? (Entrevistada 1).

Outro ponto que chamou a atenção durante as entrevistas foi a postura de acolhimento, atenção e cuidado, muito valorizada pelas mães. Tais princípios comportamentais representam uma consequência da empatia destacada anteriormente, pois o trabalho odontológico, assim como outros serviços de saúde, requer o respeito à intimidade do paciente e a confiança para a colaboração entre o dentista e o paciente e, no caso da odontopediatria, entre o profissional e o responsável. Segundo as mães consultadas, essas características são mais frequentes no atendimento prestado no ambulatório da EBMSP do que o prestado em clínicas da iniciativa privada:

Se os dentistas quando saíssem da faculdade fossem do mesmo jeito que eles são aqui, já melhoraria muito a situação que a gente ver ai fora, pois a maioria são muito ignorantes e não explicam as coisas direito, então o carinho e a atenção não podem ser esquecidos e apenas usado enquanto tá na faculdade (Entrevistada 2).

Segundo as mães, durante as várias consultas, constrói-se um vínculo entre paciente e profissional que é de extrema importância. Para tanto, é preciso que todo o processo desenvolva-se de maneira mais honesta e clara possível, para que haja confiança e fidelidade entre ambos, por isso o detentor do conhecimento técnico e científico deve explicar cada procedimento. Dessa forma, torna-se possível a extensão do tratamento para fora das portas do ambulatório: através desse tipo de comunicação, o dentista se faz presente na vida dos seus pacientes, como indicou uma das mães

$\mathrm{Eu}$ gosto muito que me explique o que eu tenho que fazer e como vai ser feito, isso cativa o paciente e ele não vai desconfiar de nada e vai aceitar o que eles falarem (Entrevistada 3). 


\section{Pontos negativos (fragilidades) no atendimento}

Em uma das entrevistas podemos é possível identificar falhas antes que inicie o tratamento, pois as mães relataram que as informações não são passadas com clareza, gerando transtornos e dificultando a abertura do prontuário, fazendo com que postergue o tratamento, como podemos identificar no trecho da entrevista:

Eu só tenho uma queixinha dos seguranças lá da portaria, do principio, lá do inicio eles fazem muita barreira e dificulta a comunicação e o acesso, as informações eles não falam direito, só chega e fala não ta marcando, e não explica quando, o porquê e o que realmente precisa trazer para o atendimento (Entrevistada 1 ).

As mães transpareceram extrema franqueza e coerência, fornecendo explicações claras sobre os seus pontos de vista, e, também, acerca dos pontos de vistas que seus filhos comunicam a elas a respeito do atendimento. Dentre os pontos negativos no atendimento apontados pelas mães, inicialmente, ressalta-se a dificuldade na marcação das consultas:

Aqui é difícil é achar vaga, principalmente eu própria que estou tentando, só que eu trouxe aquele papelzinho que dá no posto, mas não consegui porque não tinha vaga (Entrevistada 4).

Outra fragilidade exposta pelas mães foi a falta de pontualidade no atendimento, visto que as crianças não são atendidas no horário que foram marcadas:

Já peguei uns que enrolava muito, aí o trabalho não rende e isso estressa a gente, aí vai e marca, aí a gente vem com a criança pequena (Entrevistada 5).

As entrevistadas dois e seis também manifestaram seu profundo descontentamento a respeito do atraso no atendimento:

Esperamos muito para atender a gente e isso é chato, porque às vezes ficamos esperando e não atendem, aí pagamos o transporte pra não ter atendimento (Entrevistada 6).

Outra entrevistada ressaltou o mesmo problema, considerando que seu filho pode se tornar avesso ao tratamento no caso de o dentista não ser pontual. É possível que uma falha seja perdoada facilmente, porém a fala das mães aponta para uma recorrência que deve ser evitada, caso contrário, pode comprometer a própria empatia e a imagem positiva do comportamento do dentista para a criança, o que fica claro no relato a seguir:

Negativo só a demora pra atender. Meu filho nem quer vim mais, porque espera muito pra poder atender (Entrevistada 7).

\section{O dentista ideal}

A imagem do dentista ideal não transparece como unanimidade na fala das mães, porém, algumas características compartilhadas por elas devem compor aquilo que se espera de um profissional da Odontologia. As mães esperam um dentista bem qualificado, transparente quanto aos métodos empregados, e extremamente cuidadoso com o paciente. Uma mãe em especial declarou:

Quero que trate bem, que faça as coisas direitinho, mas não tenho o que falar não [dos dentistas daqui], eles são bem atenciosos com as crianças (Entrevistada 1).

No relato acima, é interessante que a entrevistada aproxima o dentista ideal do dentista real encontrado no ambulatório, destacando a postura mais humana como fator preponderante. Para essa pergunta, algumas mães foram mais objetivas e disseram apenas que esperavam que o dentista ideal "atenda bem", ou "que seja atencioso com as crianças":

Quero que trate bem. Trata meu filho muito bem, nem particular eu vejo isso, trata muito bem mesmo, com muito carinho, muita educação, demais (Entrevistada 8 ).

Deve-se ter em mente que a proposta de um dentista ideal, seja qual for, talvez seja, em sua totalidade, inalcançável, considerando a condição humana que leva a falhas. Porém, as percepções das mães foram muito enriquecedoras nesse sentido.

\section{Discussão}

Do ponto de vista da caracterização da amostra deste estudo, as mães participantes contemplaram diferentes faixas etárias, diversos níveis de escolaridade e distintos níveis salariais e motivos da consulta, o que pode revelar um caráter não tendencioso da investigação.

Considerando as percepções das mães sobre as potencialidades no atendimento da EBMSP, no serviço de odontopediatria, as mães mostraram-se satisfeitas e valorizaram, sobretudo, as questões interpessoais na relação dentista-paciente. A construção e a manutenção de laços afetivos entre o dentista e a criança representa o principal aspecto para $o$ sucesso do tratamento, segundo as mães. Cabe destacar que, em geral, as mães também valorizavam a capacidade técnica dos alunos, mas ressaltaram a importância do vínculo e da comunicação qualificada durante $o$ atendimento.

Tais aspectos também foram observados em pesquisas de percepções de mães de pacientes odontopediátricos em outras regiões e países. No caso do ambulatório da Universidade Federal de Santa Catarina, Robles et al. ${ }^{4}(2008)$ verificaram que o fornecimento de informações, o carinho, a atenção e a comunicação entre os alunos e os pacientes responsáveis, assim como a resolução dos problemas 
bucais proporcionam um atendimento que satisfaz, tanto as mães, quanto as crianças atendidas.

Para Freeman $^{1}$ (1999), é fundamental que, durante $o$ atendimento, o dentista deve ser percebido como um pai cuidadoso para com a criança, pois não se trata de um paciente qualquer, pois possíveis traumas de um tratamento desprovido de acolhimento especial podem favorecer ao desenvolvimento de medo em relação ao tratamento odontológico no futuro. Pesquisadores holandeses ressaltaram que tais aspectos se acentuam ainda mais no depoimento de mães jovens ${ }^{8}$.

As entrevistadas opinaram unanimemente que a afetividade constitui um ponto benéfico do atendimento no ambulatório odontológico da EBMSP. Esse resultado é consonante com a pesquisa de Cardoso e Grosseman $^{2}$ (2007) sobre as percepções das mães a respeito do atendimento pediátrico no ambulatório de pediatria do Hospital Universitário da Universidade Federal de Santa Catarina, ressaltando que:

Na percepção sobre o atendimento pelos estudantes, a maioria das entrevistadas estava satisfeita de forma geral, e estes aspectos foram ainda mais ressaltados, sendo os mais frequentemente mencionados: atenção dispensada, paciência, tranquilidade e carinho demonstrado pelos estudantes [...] (Ibidem, p. 36).

Ao investigar-se os pontos negativos no atendimento de odontopediatria da EBMSP, chamam a atenção questões relacionadas ao acolhimento problemas de comunicação na entrada da instituição, dificuldades na marcação de consultas, atrasos no atendimento - grande parte da insatisfação voltada para o ambiente da "sala de espera".

Da Costa et al. ${ }^{8}$ (2012) trazem considerações a respeito de uma pesquisa de satisfação com o atendimento em Fonoaudiologia, entre as quais destacam o acolhimento como elemento prioritário, pois esse melhora a comunicação, tornando o paciente mais capaz para expressar suas necessidades, garantindo que o profissional da saúde possa compreendê-las melhor e, com isso, possa estabelecer, assim, um elo de cumplicidade com o paciente. Considerando-se que se trata de crianças e de adolescentes, esse pressuposto ganha contornos especiais, quando aplicado à odontopediatria.

A compreensão do acolhimento não pode se restringir ao atendimento na cadeira do dentista, mas deve estar presente em todos os ambientes intermediários, principalmente no momento da espera pela consulta. Nesse sentido, Pimentel et al. ${ }^{9}(2011)$ destacam que:

[...] as atividades da sala de espera são direcionadas frequentemente à educação em saúde. Equipes de enfermagem e psicólogos costumam utilizar esse ambiente para divulgar programas de saúde, tirar dúvidas, criar vínculos com os usuários, ajudando no desenvolvimento do acolhimento.

O papel educador do cirurgião-dentista, reconhecido em vários depoimentos das mães como manifestação de qualidade, deve ser visto como um dos pilares do acolhimento na promoção de saúde aos pacientes. Segundo Guerrero et al. ${ }^{10}(2013)$, os profissionais de saúde e os funcionários do Sistema Único de Sáude (SUS), em geral, adotam a atitude de acolhimento, informando aos usuários sobre os serviços de saúde, bem como a forma de funcionamento desse Sistema. Uma vez que o paciente seja educado, reações decorrentes de mal-entendidos e de raiva são evitadas.

De acordo com esse estudo, um dentista ideal, na percepção das mães entrevistadas, é aquele que tem atenção com os pacientes - valorizando, assim, a relação interpessoal -, que executa o procedimento de forma correta e que, além disso, educa as mães e as crianças, em cada uma das etapas do atendimento.

Em compensação, deve-se reconhecer que o "dentista ideal" ou o "tratamento ideal" é, na prática, um pressuposto irrealizável ${ }^{14}$. Apesar disso, torna- se necessário para orientar o melhor tratamento possível aos pacientes. Ståhlnacke et al. ${ }^{12}(2007)$ destacam, como na Suécia, a pesquisa pelo conceito de dentista ideal, realizada em 1942, consuziu a um aumento da satisfação dos pacientes suecos nas décadas seguintes, atingindo um patamar mais alto nos anos de 1990, em todos os níveis, em comparação com data anterior.

Concorda-se, na presente pesquisa, com a consideração de Robles et al. ${ }^{4}$ (2008), no sentido de interpretar as percepções das mães sobre o dentista ideal com o tratamento odontopediátrico, como um cumprimento das competências da formação em nível superior em Odontologia, definidas pelo currículo, em seu artigo ${ }^{13,15}$. Essas competências dizem respeito às cognitivas (capacitação técnica avaliada no momento e após o tratamento), às afetivas (que dizem respeito ao acolhimento em suas diversas manifestações para com o paciente) e às de dimensão psicomotora (presente no bom manuseio dos materiais, jamais questionado pelas mães entrevistadas).

Em relação ao "dentista ideal", é preciso entender quais são as prioridades desejadas pelos pacientes, nesse caso, através da interlocução de suas mães. O estudo de Al-Mudaf et al. ${ }^{15}$ (2003), apesar de ter um caráter mais quantitativo, demonstrou que o perfil do dentista ideal varia conforme as faixas etárias ${ }^{15}$.

De acordo com Crossley et al. ${ }^{16}$ (2001), a definição dessas prioridades, a partir das pesquisas de satisfação, possibilitaram uma reorganização do orçamento do ambulatório da faculdade do New Hampshire, nos Estados Unidos. Essa reorientação é fundamental, porém, não deve ficar restrita ao financiamento do tratamento, assim como também deve ocorrer em todo o processo de atendimento, adequando a oferta dos serviços de saúde bucal às demandas apresentadas pela comunidade. Já Franco e Campos ${ }^{17}$ (1998) chamam a atenção para o fato de, mesmo na medicina, os pacientes terem sempre em mente um profissional ideal com o qual interagem ${ }^{17}$. 
Foi interessante constatar que, muitas vezes, as próprias entrevistadas, quando questionadas sobre o que consideravam importante para um tratamento, apresentavam-se tão satisfeitas com o tratamento que desejavam que o dentista ideal, nos consultórios públicos e particulares, agisse como os estudantes de Odontologia da EBMSP, isso é, esperavam que os alunos mantivessem, ao longo de sua carreira profissional, os pontos positivos apresentados no tratamento. Esse fato simboliza uma visão negativa dos dentistas profissionais.

Estudos sobre as percepções da satisfação a respeito do atendimento odontológico são escassos. No entanto, acredita-se que a compreensão das percepções das mães de pacientes odontopediátricos constitui um passo importante para a melhoria da saúde bucal infantil, a qual não apenas necessita de uma abordagem tecnicista, mas também de um trato humanístico, incluído nas competências e habilidades desejadas de um dentista, favorecendo diretamente a promoção de saúde desse grupo populacional. Pesquisas dessa natureza podem contribuir diretamente para a melhoria da assistência nos serviços públicos, privados e de instituições de ensino.

\section{Conclusões}

Esta pesquisa revelou que as mães encontram-se, em geral, satisfeitas com o tratamento oferecido no ambulatório de odontopediatria da EBMSP, o qual apresenta competências cognitivas (conhecimento dos procedimentos e recursos empregados), afetivas (através da atenção e transparência junto aos pacientes e suas mães) e psicomotoras (satisfação sobre a qualidade das intervenções). Contudo, a maioria das entrevistadas ressaltou a importância de aspectos psicossociais, como a presença de afetividade, de carinho e de atenção na relação dentista paciente para o sucesso do tratamento odontológico. As mães também destacaram pontos negativos no atendimento odontológico. O principal deles refere-se aos atrasos de alguns estudantes de Odontologia no horário das consultas e de problemas na marcação de consulta com os funcionários da Instituição. Esses aspectos, referentes ao acolhimento, podem comprometer, caso não sejam sanados, o padrão de satisfação estabelecido.

Espera-se, com o artigo apresentado, que a instituição continue investido recursos financeiros e pedagógicos nos pontos positivos do tratamento, mas que também elabore providências para reverter os pontos negativos, aproximando-se ainda mais da formação do "dentista ideal".

\section{Abstract}

Beside the technical experiments, Dentistry requires that research on patient satisfaction contribute to the advancement of knowledge and health services, especially in the juvenile group, stage of life very important in determining the quality of life related oral health. Objective: This study aims to describe the perceptions of mothers on dental care provided in Clinical Child II of Bahian School of Medicine and Public Health (EB$M S P)$, by curriculum component of pediatric dentistry. Subjects and methods: This paper will be a qualitative study conducted with mothers of children seen in the outpatient EBMSP. The sample is convenience: about 10 mothers who will be interviewed individually until reaching a saturation point in the interviews that follow a semi-structured categories that address related quality of services offered in order to identify their weaknesses and potential, the skills (cognitive, technical, socio-emotional. Results: Cognitive, affective and psychomotor aspects were highlighted in attendance, although psychosocial were most valued by mothers; Problems in the host were characterized by difficulty in setting up appointments and tardiness of students; stated ideal dentist who met different skills, beyond technical. Conclusions:Perceptions of outpatient care may contribute to improving the quality of services offered, as well as the oral health of the assisted population.

Keywords: Dental care Children.Pediatric dentistry.

\section{Referências}

1. Freeman R. A psychodynamic understanding of the dentist-patient interaction. British Dental Journal 1999; 186(10):503-6

2. Cardoso PM, Grosseman S, Robles ACC. Percepção de mães sobre o atendimento prestado a seus filhos por acadêmicos da $11^{\mathrm{a}}$ fase em um ambulatório de pediatria. Arquivos Catarinenses de Medicina 2007; 36(3):42-51.

3. Silva RVD, Muniz L. Clareamento externo para dentes com calcificação distrófica da polpa: relato de caso clínico. R Ci Méd Biol 2007; 6(2):247-51.

4. Robles ACC, Grosseman S, Bosco VL. Satisfação com o atendimento odontológico: estudo qualitativo com mães de crianças atendidas na Universidade Federal de Santa Catarina. Ciênc Saúde Coletiva 2008; 13(1):43-9.

5. Jung HP, Van Horne F, Wensing M, Hearnshaw H, Grol R. Which aspects of general practitioners' behavior determine patients' evaluations of care?. Soc Sci Med 1998; 47 (8): 1077-87.

6. Conselho Nacional de Saúde. Resolução nํㅜ 196, de 10 de outubro de 1996. Conselho Nacional de Saúde. Brasil, Brasília, 10 de outubro de 1996, seção 1, p. 21082

7. Conselho Federal de Odontologia. Resolução n. 118, de 11 de maio de 2012. Diário Oficial da Republica Federativa do Brasil, Brasília, 11 de maio 2012, seção 1, p. 118.

8. Da Costa JC, Giustti SA, Murofuse IS, Gumz AL. Acesso ao serviço de fonoaudiologia: a implantação do acolhimento no município de Toledo - PR. Rev CEFAC 2012; 14(5): 977-83.

9. Pimentel AF, Barbosa RM, Chagas M. A musicoterapia na sala de espera de uma unidade básica de saúde: assistência, autonomia e protagonismo. Interface (Botucatu) 2011; 15(38): 741-54. 
10. Guerrero P, De Mello ALSF, De Andrade SR, Erdmann AL. User embracement as a good practice in primary health care. Texto Contexto-Enferm 2013; 22 (1): 132-40.

11. Lima MADS, Ramos DD, Rosa RB, Nauderer TM, Davis R. Acesso e acolhimento em unidades de saúde na visão dos usuários. Acta Paul Enferm 2007; 20 (1): 12-77

12. Ståhlnacke K, Söderfeldt B, Unell L, Halling A, Axtelius B. Perceived oral health: changes over 5 years in one Swedish age-cohort. Community Dent Oral Epidemiol 2003; 31 (4): 292-9.

13. Brasil. Resolução CNE/CES 3, de 19 de fevereiro de 2002. Diário Oficial da União, Brasil, Brasília, 4 de mar 2002, seção 1, p. 10.

14. Morita MC, Kriger L. Mudanças nos cursos de Odontologia e a interação com o SUS. Revista da ABENO 2003; 4(1): 17-21.

15. Al-Mudaf BA, Moussa MAA, Al-Terky MA, Al-Dakhil GD, El-Farargy AE, Al-Ouzairi SS. Patient Satisfaction with Three Dental Speciality Services: A Centre-Based Study. Med Princ Pract 2003; 12 (1): 39-43.

16. Crossley ML, Blinkhorn A, Cox M. 'What do our patients really want from us?': Investigating patients perceptions of the validity of the Chartermark criteria. Br Dent J 2001; 190 (11): 602-6.

17. Franco SC, Campos GWS. Avaliação da qualidade de atendimento ambulatorial em pediatria em um hospital universitário. Cad Saúde Públ 1998; 14 (1): 61-70.

\section{Endereço para correspondência:}

Tatiana Frederico de Almeida

Rua João Bião de Cerqueira 251, Pituba

41.830.580 Salvador-BA

Fone: (71) 3351-4447/9933-4886

E-mail: tatifrederico@yahoo.com.br

Recebido: 02/01/2014. Aceito: 17/08/2014. 